\title{
Resistance training alone or combined with leucine supplementation improves the serum lipid profile of diabetic rats, whereas leucine alone does not
}

Carlos Eduardo C. Martins, Vanessa B. de Sousa Lima, Henrique Quintas T. Ribeiro, Julio Tira pegui

\author{
Department of Food \& Experimental Nutrition, Faculty of Pharmaceutical Sciences, \\ University of Sao Paulo, Sao Paulo, SP, Brazil \\ E-mail:martinscec@usp.br
}

\begin{abstract}
Objectives. Diabetes mellitus is associated with dyslipidemia, which contributes to a higher risk of thrombosis, atherosclerosis and cardiovascular disease. This study evaluated the effects of leucine and resistance training on the serum lipid profile in rats with streptozotocin-induced diabetes for 8 weeks.

Methods. Wistar rats with neonatal streptozotocin-induced diabetes were treated with leucine supplementation (5\%) and/or resistance training (3 days per week) for 8 weeks, and divided in DL (diabetic and leucine), DT (diabetic and resistance training group) and DLT (diabetic, leucine and resistance training) groups. Others 2 groups of animals received isonitrogen AIN-93M diet that was defined as a control diet: group D (diabetic untreated) and group C (non-diabetic).

Results. The decrease in serum total cholesterol and increase in high-density lipoprotein cholesterol (HDL-C) was observed in the resistance training-induced diabetic rats when compared with diabetic rats. There was no change in serum lipid profile in leucine-supplemented diabetic rats and no synergistic effect of leucine and resistance training. The fasting glucose levels were reduced in all animals treated compared to $\mathrm{D}$ group.

Conclusion. The diabetic trained rats demonstrate a protective effect of resistance training on the serum lipid profile.
\end{abstract}

Key words: resistance training, leucine, diabetes mellitus, rats, lipid profile

Diabetes mellitus (DM) is defined as a group of metabolic diseases with the presence of a hyperglycemic state due to impairments in insulin release or function in type 1 and type $2 \mathrm{DM}$, respectively (D'Souza et al. 2013). The development of each form of diabetes drastically differs, but the resulting pathologies often overlap. In both types of diabetes, alterations occur in the metabolism of lipids and lipoproteins. This characteristic dyslipidemia of DM is associated with an increased oxidative stress and has been implicated in the pathogenesis of the atherothrombotic macrovascular disease (Bierman et al.
1966). Hypercholesterolemia in streptozotocin-induced (STZ) diabetic rats results from the increased synthesis of cholesterol-ester rich lipoproteins and from the inhibited removal of circulating low-density lipoprotein cholesterol (LDL-C) (Mathe 1995).

Leucine supplementation has potential therapeutic effects on body composition and improves the glucose and cholesterol metabolism in animal models (Donato et al. 2006; Zhang 2007; Liu et al. 2014). The leucine may also alter serum lipid profile in experimental obesity models. The leucine supplementation reduced the serum concentration of total cholesterol

Corresponding author: C.E.C. Martins, Department of Food \& Experimental Nutrition, Faculty of Pharmaceutical Sciences, University of Sao Paulo, Av. Prof. Lineu Prestes 580, Block 14, CEP 05389-970, Sao Paulo, SP, Brazil; phone: (011) 3091-3309; e-mail: martinscec@usp.br. 
and this response did not depend on body weight or fat mass changes (Torres-Leal et al. 2011). Zhang et al. (2007) have observed that a reduction in cholesterol levels with leucine supplementation in obese mice was independent of leucine-induced changes in adiposity. However, little is known about the molecular mechanisms that are activated by leucine and that change cholesterol metabolism. It is possible that this amino acid stimulates an increase of fatty acid synthesis in the muscle accompanied by a concomitant decrease in the liver fatty acid synthesis considered to be a positive effect on lipid metabolism (Morifuji et al. 2005).

In addition, the resistance training has also been considered as a potent non-pharmacological tool. In STZ-induced diabetic rats, resistance training increased muscle strength, interleukin-15 (IL-15) secretion capacity as a myokine that is not depressed in the diabetic state and improved adiposity and obesityassociated inflammation and the serum lipid profile (Molanouri et al. 2014; Safarzade and Talebi-Garakani 2014). Number of studies regarding the effects of resistance training on lipoproteins metabolism in diabetes is very limited and their findings are still controversial. Research show that resistance training associated with aerobic training increases high-density lipoprotein cholesterol (HDL-C) and reduces LDL-C (Safarzade and Talebi-Garakani 2014). Furthermore, studies in humans show that resistance training alone reduces or does not alter the concentrations of LDLC in blood (Cauza et al. 2005; Ghanbari -Niaki et al. 2011) and decreases or increases circulating HDL-C (Costa et al. 2011; Williams et al. 2011). Despite the disagreements, Fahlman et al. (2002) have reported that the resistance training alone is able to alter the metabolism of lipoproteins, which may help in the prevention of cardiovascular and atherosclerotic diseases.

We investigated the effects of two therapeutic strategies on the serum lipid profile of STZ-induced diabetic rats, i.e. a resistance training program and leucine supplementation. To our knowledge, this is the first report evaluating the effects of combined interventions on the serum lipid profile in STZ-induced diabetic rats.

\section{Materials and methods}

Animals. Five-day-old male Wistar rats (40 animals in total) were separated from their mothers for $8 \mathrm{~h}$ and divided into two groups: a diabetic group $(n=32)$ that received an intraperitoneal (i.p.) injection of STZ (120 mg/kg body weight) freshly diluted in ci- trate buffer (10 mM Na citrate, $\mathrm{pH} 4.5)$ and a non-diabetic group $(n=8)$ that received only the citrate buffer injection (i.p.) in an equivalent volume and served as the control group (C group) (Takada et al. 2007; Martins et al. 2017). Twenty-one days after birth (at weaning), all of the rats from the diabetic group presented glycemia above $150 \mathrm{mg} / \mathrm{dl}$ and were included in this study, and were divided into four groups: the D group $(n=8)$ received an isonitrogen AIN-93M diet that was defined as control diet; the DT group $(n=8)$ received the same isonitrogen diet and performed resistance training; the DL group $(\mathrm{n}=8)$ was treated diet supplemented with 5\% L-leucine; and the DLT group $(n=8)$ was treated with $5 \%$ of L-leucine and resistance training. The interventions were performed for 8 weeks. The animals were treated already at 21 days of age because it is time of weaning of the animals, which begin to feed chow and was possible to analyze the glycemia in tail of rats. At the end of last week of intervention, the animals were euthanized by decapitation after $6 \mathrm{~h}$ of fasting.

Experimental design. The experimental diets were prepared according to the recommendations of the American Institute of Nutrition for adult rats (AIN-93M diet). Based in this diet, $50 \mathrm{~g}$ of cornstarch $/ \mathrm{kg}$ of chow were replaced by $50 \mathrm{~g}$ of leucine (diet supplemented with 5\% L-leucine) or by $50 \mathrm{~g}$ of a non-essential amino acid mix (alanine, aspartic acid, glycine, proline and serine) with equivalent quantities of nitrogen of diet supplemented with leucine (isonitrogen diet) (Reeves et al. 1993).

The exercise consisted of climbing a ladder inclined at $85^{\circ}$ nine times. Training sessions were held three times a week in the morning. This protocol provides an animal model of resistance exercise that closely resembles the exercise parameters and physiological adaptations observed in humans who participate in resistance training (Hornberger and Farrar 2004). All of the methods used were approved by the Local Ethical Committee on Animal Use according to the Brazilian College of Animal Experimentation (protocol: 14.2014-P451).

Measurement of concentrations of serum lipoproteins and serum fasting glucose. The concentrations of serum lipoproteins [total cholesterol (TC), triglycerides (TG), HDL-C, LDL-C] were measured by enzymatic methods using a Labtest ${ }^{\oplus}$ kit Labtest $^{\circ}$ diagnostica AS, Brazil). The very low-density lipoprotein cholesterol (VLDL-C) was calculated using Friedewald's equation as follows: VLDL-C $=$ TG/5. The antiatherogenic index (AAI) was calculated using the formula (AAI $=$ HDL-C $\times 100 /$ TC - HDL-C). This index has an inverse relationship with coronary heart 
disease risk by lipoprotein abnormalities that are potentially atherogenic (Guido and Joseph 1992).

Serum glucose was enzymatically determined using the following commercial kits. Glucose (Labtest ${ }^{\circledR}$ diagnostica AS, Brazil).

Statistical analysis. The results are expressed as the mean \pm SEM. The dependent variables were tested by one-way followed by Tukey's test (GraphPad Software Inc., San Diego, Calif., USA). Differences were considered significant at $\mathrm{p}<0.05$.

\section{Results}

Lipid profile. Serum TC was greater in the D group compared with the $\mathrm{C}$ group $(\mathrm{p}<0.05)$. The serum TC in the diabetic group was reduced only in the trained groups (DT and DLT) in relation to D group. HDL-C was significantly higher in the DT and DLT groups compared to the $\mathrm{D}$ group $(\mathrm{p}<0.05)$. According to the AAI, DT and DLT groups were higher than the $\mathrm{C}, \mathrm{D}$ and DL groups $(\mathrm{p}<0.05)$ and may also be related to the antiatherogenic properties of HDL-C.

No differences were observed in the TC, TG, HDLC, LDL-C, VLDL-C, and AAI values in the D and DL groups ( $>0.05)$, which shows that leucine supplementation alone could not modulate the serum lipid profile. This difference in results may be caused by the use of a diabetic and non-obese model in our study.

Fasting glucose. Figure 1 shows the lower fasting serum glucose in the groups treated with resistance training alone or combined with a leucine (DT and DLT groups), and DL group compared to the D group $(\mathrm{p}<0.05)$.

\section{Discussion}

The diabetic untreated group of rats showed higher TC, which can be explained by insulin pathway. Insulin also plays an important role in the metabolism of lipids. Under normal condition, insulin increases the receptor-mediated removal of LDL-C (Saravanan and Pari 2005) and decreases the activity or secretion of insulin during diabetes causes hypercholesterolemia (Saravanan and Pari 2005; Gengiah et al. 2014). However, the groups that were submitted to resistance training had a reduction in TC. Similar results have been observed in type I diabetic men subjected to resistance training (Durak et al. 1990). Lira et al. (2010) have shown that low/moderate or high-intensity resistance training improvement the lipid profile of untrained males, although the mechanisms underlying this effect are unclear. The authors speculated that the reduction in $\mathrm{TC}$ is a result of the exchange of cholesterol ester between tissues and lipoproteins to HDL-C. These mechanisms include increases in lecithin cholesterol acyltransferase (LCAT), the enzyme that is responsible for cholesterol ester transfer to HDL-C. LCAT levels have been shown to be increased following exercise training (Nicastro et al. 2012).

The groups that were submitted to resistance training also had increased HDL-C concentrations, which also support a model of diabetes induced by STZ with resistance training (Nicastro et al. 2012; Molanouri et al. 2014). These TC and HDL-C data confirm the beneficial effect of resistance training on the serum lipid profile, which may have a protective effect on the cardiovascular diseases, according to the AAI (Kraus et al. 2002; Williams et al. 2007). In these trained groups the AAI also proved to be high and may be related to anti-atherogenic properties of HDL-C. In addition, HDL-C transports circulating cholesterol and delivers it to the liver. This lipoprotein also has other atheroprotective functions and anti-inflammatory, antioxidant, and vasodilator properties. Normal functional HDL-C has high levels of antioxidants and redox-active proteins (Podrez 2010).

Some studies have reported that leucine supplementation for 6 weeks reduces the serum TC, but not TG and HDL-C (Torres-Leal et al. 2011), and 14 weeks of leucine supplementation in drinking water decreases the TC and LDL-C in obese animals induced by a high-fat diet (Zhang et al. 2007). However, in our study, no differences were observed in the TC, TG, HDL-C, LDL-C, VLDL-C and AAI values in the $\mathrm{D}$ and DL groups ( $\mathrm{p}>0.05)$, which indicates that leucine supplementation alone could not modulate the serum lipid profile.

Resistance training was also able to reduce serum fasting glucose. This result is associated with the fact that resistance training enhances the blood glucose uptake by skeletal muscle through the insulindependent mechanisms. The exercise increases the potential for the translocation of GLUT-4 into the membrane of skeletal muscle cells and enhances the skeletal muscle and whole-body insulin sensitivity and insulin-stimulated glucose uptake can persist up to 24-48 hours after one exercise session (Holten et al. 2004; Cartee 2015).

The improvement of circulating glucose levels in the DL group may be related to the action of leucine in the recovery of insulin signaling at long term (Hinault et al. 2006). In study of Liu et al. (2014), the insulin-stimulated glucose uptake and Akt phosphorylation were inhibited in skeletal muscle cells by the wortmannin, but the inhibition was partially 

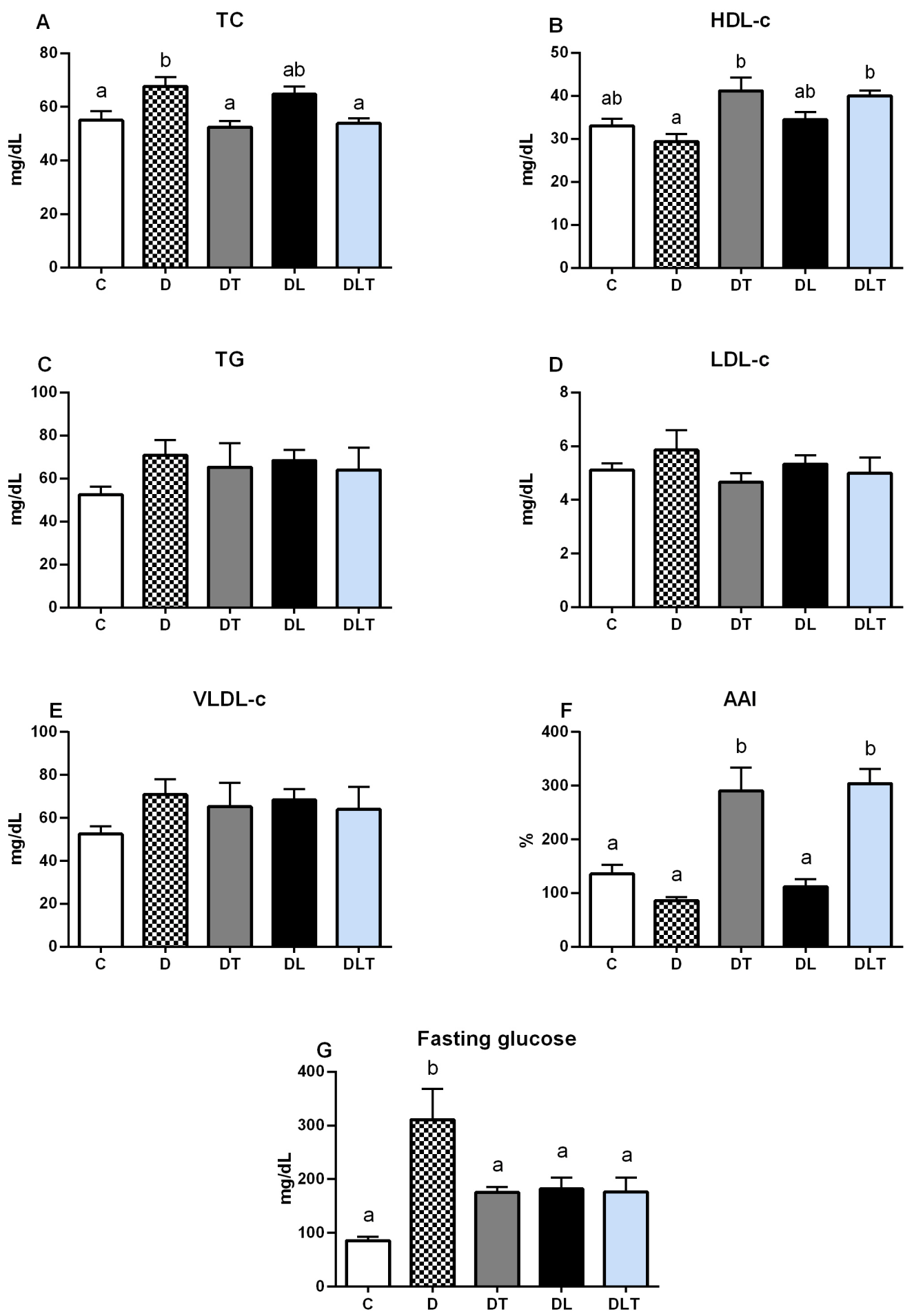

Figure 1. Serum lipid profile and fasting glucose of streptozotocin-induced treated rats submitted to leucine supplementation and resistance training. Data are means \pm SEM. Note that above each bar, there are letters. Bars sharing the same letters do not differ statistically. Letters not shared indicate statistically significant differences $(\mathrm{p}<0.05)$. Abbreviations: TC - total cholesterol; TG - triglycerides; HDL-C - high-density lipoprotein; LDL-C - low-density lipoprotein; VLDL-C - very low-density lipoprotein; AAI - antiatherogenic index; C - Control group; D - diabetic group; DT - diabetic and resistance training group; DL - diabetic and leucine; DLT - diabetic, leucine and resistance training. 
reversed by leucine. In addition, this recovery of the leucine-induced Akt phosphorylation was neutralized by knocking down of the mammalian target of rapamycin complex 2 (mTORC2) with specific siRNA. These findings show that leucine facilitate the insulin signaling and insulin-induced glucose uptake in skeletal muscle cells through both mTORC1 and mTORC2, implicating the positive effect of this amino acid on glycemia control in diabetics.

\section{Conclusions}

Leucine supplementation alone did not promote significant improvements in the serum lipid profile. However, resistance training alone or combined with leucine supplementation increased the HDL-C and $\mathrm{AAI}$ and reduced the TC in the serum of our experimental model of diabetes, improving dyslipidemia and promoting cardiovascular benefits in these animal models, but without any synergistic effect of leucine and resistance training.

\section{Acknowledgements}

CECM, VBSL and HQTR were the principal investigator of this study. JT supervised this work. All the authors participated in the clinical research. CECM, VBSL and HQTR are supported by CNPQ and CAPES (Grants 134442/2013-0, 159049/2012-1). This study was supported by FAPESP (2012/21087-4).

\section{References}

Bierman EL, Amaral JA, Belknap BH. Hyperlipidemia and diabetes mellitus. Diabetes 15, 675-679, 1966.

Cartee GD. Mechanisms for greater insulin-stimulated glucose uptake in normal and insulin resistant skeletal muscle after acute exercise. Am J Physiol Endocrinol Metab 309, E949-E959, 2015.

Cauza E, Hanusch-Enserer U, Strasser B, Ludvik B, Metz-Schimmerl S, Pacini G, Wagner O, Georg P, Prager R, Kostner K, Dunky A, Haber P. The relative benefits of endurance and strength training on the metabolic factors and muscle function of people with type 2 diabetes mellitus. Arch Phys Med Rehabil 86, 1527-1533, 2005.

Costa RR, Lima AC, Tagliari M, Martins KLF. Effects of resistance training on the lipid profile in obese women. J Sports Med Phys Fitness 51, 169-177, 2011.

D'Souza DM, Al-Sajee D, Hawke TJ. Diabetic myopathy: impact of diabetes mellitus on skeletal muscle progenitor cells. Front Physiol 20, 379, 2013.

Donato JJr, Pedrosa RG, Cruzat VF, Pires IS, Tirapegui J. Effects of leucine supplementation on the body composition and protein status of rats submitted to food restriction. Nutrition 22, 520-527, 2006.

Durak EP, Jovanovic-Peterson L, Peterson CM. Randomized crossover study of effect of resistance training on glycemic control, muscular strength, and cholesterol in type I diabetic men. Diabetes Care 13, 1039-1043, 1990.

Fahlman MM, Boardley D, Lambert CP, Flynn MG. Effects of endurance training and resistance training on plasma lipoprotein profiles in elderly women. J Gerontol A Biol Sci Med Sci 57, B54-B60, 2002.

Gengiah K, Hari R, Anbu J. Antidiabetic antihyperlipidemic and hepato-protective effect of Gluconorm-5: A polyherbal formulation in steptozotocin induced hyperglycemic rats. Anc Sci Life 34, 23-32, 2014.

Ghanbari-Niaki A, Saghebjoo M, Hedayati M. A single session of circuit-resistance exercise effects on human peripheral blood lymphocyte ABCA1 expression and plasma HDL-C level. Regul Pept 166, 42-47, 2011.

Guido S, Joseph T. Effect of chemically different calcium antagonists on lipid profile in rats fed on a high fat diet. Indian J Exp Biol 30, 292-294, 1992.

Hinault C, Mothe-Satney I, Gautier N, Van Obberghen E. Amino acids require glucose to enhance, through phosphoinositide-dependent protein kinase 1, the insulin-activated protein kinase B cascade on insulin-resistant adipocytes. Diabetologia 49, 1017-1026, 2006.

Holten MK, Zacho M, Gaster M, Juel C, Wojtaszewski JF, Dela F. Strength training increases insulin-mediated glucose uptake, GLUT4 content, and insulin signaling in skeletal muscle in patients with type 2 diabetes. Diabetes 53, 294-305, 2004.

Hornberger TAJr, Farrar RP. Physiological hypertrophy of the FHL muscle following 8 weeks of progressive resistance exercise in the rat. Can J Appl Physiol 29, 16-31, 2004.

Kraus WE, Houmard JA, Duscha BD, Knetzger KJ, Wharton MB, McCartney JS, Bales CW, Henes S, Samsa GP, Otvos JD, Kulkarni KR, Slentz CA. Effects of the amount and intensity of exercise on plasma lipoproteins. N Engl J Med 347, 1483-1492, 2002.

Lira FS, Yamashita AS, Uchida MC, Zanchi NE, Gualano B, Martins EJr, Caperuto EC, Seelaender M. Low and moderate, rather than high intensity strength exercise induces benefit regarding plasma lipid profile. Diabetol Metab Syndr 2, 31, 2010. 
Liu H, Liu R, Xiong Y, Li X, Wang X, Ma Y, Guo H, Hao L, Yao P, Liu L, Wang D, Yang X. Leucine facilitates the insulin-stimulated glucose uptake and insulin signaling in skeletal muscle cells: involving mTORC1 and mTORC2. Amino Acids 46, 1971-1979, 2014.

Martins CEC, Lima VBS, Schoenfeld BJ, Tirapegui J. Effects of leucine supplementation and resistance training on myopathy of diabetic rats. Physiol Rep 5, e13273, 2017.

Mathe D. Dyslipidemia and diabetes: animal models. Diabete Metab 21,106-111, 1995.

Molanouri SM, Hassan ZM, Quinn LS, Gharakhanlou R, Baghersad L, Mahdavi M. Time course of IL-15 expression after acute resistance exercise in trained rats: effect of diabetes and skeletal muscle phenotype. Endocrine 49, 396-403, 2014.

Morifuji M, Sakai K, Sanbongi C, Sugiura K. Dietary whey protein downregulates fatty acid synthesis in the liver, but upregulates it in skeletal muscle of exercise-trained rats. Nutrition 21, 1052-1058, 2005.

Nicastro H, da Luz CR, Chaves DF, das Neves W, Valente KS, Lancha AHJr. Leucine supplementation combined with resistance exercise improves the plasma lipid profile of dexamethasone-treated rats. Lipids Health Dis 11, $11: 7,2012$.

Podrez EA. Anti-oxidant properties of high-density lipoprotein and atherosclerosis. Clin Exp Pharmacol Physiol 37, 719-725, 2010.

Reeves PG, Nielsen FH, Fahey GCJr. AIN-93 purified diets for laboratory rodents: final report of the American Institute of Nutrition ad hoc writing committee on the reformulation of the AIN-76A rodent diet. J Nutr 123, 1939-1951, 1993.

Safarzade A, Talebi-Garakani E. Short term resistance training enhanced plasma apoA-I and FABP4 levels in Streptozotocin-induced diabetic rats. J Diabetes Metab Disord 13, 41, 2014.

Saravanan R, Pari L. Antihyperlipidemic and antiperoxidative effect of Diasulin, a polyherbal formulation in alloxan induced hyperglycemic rats. BMC Complement Altern Med 5, 14, 2005.

Takada J, Machado MA, Peres SB, Brito LC, Borges-Silva CN, Costa CE, Fonseca-Alaniz MH, Andreotti S, Lima FB. Neonatal streptozotocin-induced diabetes mellitus: a model of insulin resistance associated with loss of adipose mass. Metabolism 56, 977-984, 2007.

Torres-Leal FL, Fonseca-Alaniz MH, Teodoro GF, de Capitani MD, Vianna D, Pantaleao LC, Matos-Neto EM, Rogero MM, Donato JJr, Tirapegui J. Leucine supplementation improves adiponectin and total cholesterol concentrations despite the lack of changes in adiposity or glucose homeostasis in rats previously exposed to a high-fat diet. Nutr Metab (Lond) 8, 62, 2011.

Williams MA, Haskell WL, Ades PA, Amsterdam EA, Bittner V, Franklin BA, Gulanick M, Laing ST, Stewart KJ. Resistance exercise in individuals with and without cardiovascular disease: 2007 update: a scientific statement from the American Heart Association Council on Clinical Cardiology and Council on Nutrition, Physical Activity, and Metabolism. Circulation 116, 572-584, 2007.

Williams AD, Almond J, Ahuja KD, Beard DC, Robertson IK, Ball MJ. Cardiovascular and metabolic effects of community based resistance training in an older population. J Sci Med Sport 14, 331-337, 2011.

Zhang Y, Guo K, LeBlanc RE, Loh D, Schwartz GJ, Yu YH. Increasing dietary leucine intake reduces diet-induced obesity and improves glucose and cholesterol metabolism in mice via multimechanisms. Diabetes 56, 1647$1654,2007$. 